\title{
Narrowband Angle of Arrival Estimation Exploiting Graph Topology and Graph Signals
}

\author{
Ian K. Proudler, Vladimir Stankovic, and Stephan Weiss \\ Department of Electronic \& Electrical Engineering, University of Strathclyde, Glasgow G1 1XW, Scotland \\ \{ian.proudler,vladimir.stankovic,stephan.weiss\}@ strath.ac.uk
}

\begin{abstract}
Based on recent results of applying graph signal processing (GSP) to narrowband angle of arrival estimation for uniform linear arrays, we generalise the analysis to the case of arrays with elements placed arbitrarily in three dimensional space. We comment on the selection of the adjacency matrix, analyse how this new approach compares to the multiple signal classification (MUSIC) algorithm, and provide an efficient implementation. We demonstrate that the GSP approach can perform as well as the MUSIC algorithm in terms of accuracy and computational cost. Simulations indicate that the proposed GSP approach avoids the severe performance degradation with which MUSIC is associated at low signal to noise ratios.
\end{abstract}

\section{INTRODUCTION}

An important task in array processing is to estimate the angle of arrival (AoA) of any sources that illuminate the sensors. This is often based on the second order statistics of the array data. For example, the 'classic' multiple signal classification (MUSIC) algorithm [1] estimates the data covariance matrix; from its eigenvalue decomposition (EVD), MUSIC then derives a subspace decomposition that enables the AoA estimation. More than three decades after its publication, the MUSIC algorithm still remains subject to further investigations and extensions, see e.g., [2]-[4].

Although array processing algorithms such as MUSIC are not necessarily restricted to discrete data, many implementations operate on regularly spaced sampling in time and space. This however is not necessary: sampling in time can be non-uniform [5] particularly in the case of compressive sensing [6], and sampling in space can deviate from the linear uniformly spaced array (ULA), either deliberately or because of array deformation or calibration errors, to arbitrary array configurations [7], [8].

Graph signal processing (GSP) and graph spectral analysis allow the characterisation and efficient analysis of data that has been obtained on an irregularly sampled grid [10], and therefore provide an interesting fit to an array whose elements may be arbitrarily arranged in space. To date, two papers have attempted to harness GSP for array signal processing and AoA estimation in particular: [11] experimentally established a coarse correspondence of the graph Fourier transform (GFT) coefficients to the AoA for a single source in a ULA; also for a ULA, [12] have chosen the graph topology, and hence

This work was supported by the Engineering and Physical Sciences Research Council (EPSRC) Grant number EP/S000631/1 and the MOD University Defence Research Collaboration in Signal Processing. the GFT, such that a MUSIC-like subspace projection can be exploited to estimate the AoA of a source. Specifically, the graph is constructed so that the steering vector for the source signal is an eigenvector (with unit eigenvalue) of the graph's adjacency matrix. Hence, in the noise-free case, the array data will be orthogonal to the subspace defined by the remaining eigenvectors.

The ULA in [11], [12] defines a simple and straightforward adjacency between sensor elements, and derives a cyclic spatial graph structure by connecting each sensor node with its two nearest neighbours using unweighted [11] or weighted edges [12]. In [12], temporal samples acquired by each sensor are also modelled by a cyclic graph. Modelling the ULA by a cyclic graph leads to a sparse graph adjacency matrix [18] that contains only two non-zero elements in each row.

In this paper, we further develop the approach in [12] and particularly consider the case of non-uniform sampling, i.e. the case where arrays may be arbitrarily distributed in three dimensional space [9]. Since for an arbitrary array, spatial adjacency of sensors is not clearly defined, and sparsity of a graph's adjacency matrix has no implications on the sparsity of the GFT, we replace the adjacency matrix used in [12] for a fully connected graph in both spatial and temporal domains. The resulting algorithm has a similar philosophy to that in [12] but differs in detail. We further develop a low complexity scheme, and highlight the performance difference between the GSP and MUSIC approaches. We show that the former, including its particularisation in [12], operates as a matched subspace detector [13].

First, we provide a brief review of the array signal model and MUSIC algorithm in Sec. II. Based on this, the graph topology and the GFT are motivated in Sec. III. Our GSPbased AoA estimation approach is then analysed in Sec. IV and compared to MUSIC via simulations in Sec. V.

\section{Signal Model And Angle of ARrival Estimation}

\section{A. Signal Model}

We assume $M$ sensors located arbitrarily in space, such that $\mathbf{r}_{m} \in \mathbb{R}^{3}$ is the Cartesian coordinate vector for the $m$ th sensor, with $m=1, \ldots, M$. With respect to this array, a far-field source has a planar wavefront with normal vector $\mathbf{k}_{\varphi, \vartheta}=[\sin \vartheta \cos \varphi, \sin \vartheta \sin \varphi, \cos \vartheta]^{\mathrm{T}}$ travelling across the array, whereby the source direction is given in spherical coordinates by azimuth $\varphi$ and elevation $\vartheta$. When normalised 
by the propagation speed $c$ in the medium, $\mathbf{k}_{\varphi, \vartheta} / c$ is also known as the source's slowness vector.

The time delay which the wavefront experiences at the $m$ th element relative to the origin is $t_{m}=\frac{1}{c} \mathbf{k}_{\varphi, \vartheta}^{\mathrm{T}} \mathbf{r}_{m}$. If the unit length in the coordinate system is chosen as half the minimum wavelength, then $\left|\mathbf{k}_{\varphi, \vartheta}\right|=1=\lambda_{\min } / 2=c /\left(2 f_{\max }\right)=c T_{\mathrm{s}}$, where $T_{\mathrm{s}}$ is the sampling period assuming critical sampling in time. Therefore, $t_{m}=\tau_{m} T_{\mathrm{s}}$ with

$$
\tau_{m}=\mathbf{k}_{\varphi, \vartheta}^{\mathrm{T}} \mathbf{r}_{m},
$$

which measures the wavefront's delay relative to the origin in samples.

If the array is illuminated by a narrowband source (labelled i) from direction $\left\{\varphi_{i}, \vartheta_{i}\right\}$, then its normalised angular frequency $\Omega_{i}$ turns the delay in (1) into a phase shift. The steering vector, which uniquely describes the phase shift for a source characterised by $\left\{\Omega_{i}, \varphi_{i}, \vartheta_{i}\right\}$, is

$$
\mathbf{a}_{\Omega_{i}, \varphi_{i}, \vartheta_{i}}=\frac{1}{\sqrt{M}}\left[\mathrm{e}^{\mathrm{j} \Omega_{i} \tau_{1}}, \ldots, \mathrm{e}^{\mathrm{j} \Omega_{i} \tau_{M}}\right]^{\mathrm{H}},
$$

with $\tau_{m}, m=1, \ldots, M$, depending on $\left\{\varphi_{i}, \vartheta_{i}\right\}$ via (1). Also note that with the relative time lag between the $m$ th and $\mu$ th sensor being

$$
\tau_{m, \mu}=\mathbf{k}_{\varphi_{i}, \vartheta_{i}}^{\mathrm{T}}\left(\mathbf{r}_{\mu}-\mathbf{r}_{m}\right),
$$

the corresponding phase shift between the two sensors is reflected in the complex gain $\mathrm{e}^{-\mathrm{j} \Omega_{i} \tau_{m, \mu}}$.

Using the steering vector in (2), and assuming no loss in gain across the array, the $M$ sensor signals $x_{m}[n] \in \mathbb{C}, m=$ $1, \ldots, M$ with time index $n$, can be collected into a vector $\mathbf{x}[n] \in \mathbb{C}^{M}$,

$$
\mathbf{x}[n]=\sum_{i=1}^{I} \mathbf{a}_{\Omega_{i}, \varphi_{i}, \vartheta_{i}} s_{i}[n]+\mathbf{v}[n],
$$

where $s_{i}[n], i=1, \ldots, I$, is the $i$ th of $I$ narrowband source signals illuminating the array, each with parameters $\left\{\Omega_{i}, \varphi_{i}, \vartheta_{i}\right\}$. The term $\mathbf{v}[n] \in \mathbb{C}^{M}$ in (4) represents spatially and temporally uncorrelated zero-mean Gaussian noise with $\mathcal{E}\left\{\mathbf{v}[n] \mathbf{v}^{\mathrm{H}}[n-\tau]\right\}=\sigma_{v}^{2} \mathbf{I} \delta[\tau]$, where $\mathcal{E}\{\cdot\}$ is the expectation operator, $\mathbf{I}$ is the identity matrix, and $\delta[\tau]$ the impulse function.

\section{B. Covariance Matrix and its Eigenvalue Decomposition}

For independent narrowband sources with $\Omega_{1}=\cdots=\Omega_{I}$, the second order statistics of the data are captured by the spatial covariance matrix

$$
\mathbf{R}=\mathcal{E}\left\{\mathbf{x}[n] \mathbf{x}^{\mathrm{H}}[n]\right\}=\sum_{i=1}^{I} \sigma_{i}^{2} \mathbf{a}_{\Omega_{i}, \varphi_{i}, \vartheta_{i}} \mathbf{a}_{\Omega_{i}, \varphi_{i}, \vartheta_{i}}^{\mathrm{H}}+\sigma_{v}^{2} \mathbf{I},
$$

where $\sigma_{i}^{2}$ is the power of the $i$ th source. Thus, $\mathbf{R}$ contains information on the angles of arrival $\left\{\varphi_{i}, \vartheta_{i}\right\}$ via the steering vectors $\mathbf{a}_{\Omega_{i}, \varphi_{i}, \vartheta_{i}}^{\mathrm{H}}, i=1, \ldots, I$.

The covariance matrix can be factorised via the EVD $\mathbf{R}=$ $\mathbf{Q} \mathbf{\Lambda} \mathbf{Q}^{\mathrm{H}}=\sum_{m=1}^{M} \lambda_{m} \mathbf{q}_{m} \mathbf{q}_{m}^{\mathrm{H}}$ with unitary $\mathbf{Q}=\left[\mathbf{q}_{1}, \ldots, \mathbf{q}_{M}\right]$ containing the eigenvectors and $\boldsymbol{\Lambda}=\operatorname{diag}\left\{\lambda_{1}, \ldots, \lambda_{M}\right\}$ the eigenvalues of $\mathbf{R}$. This factorisation is structurally similar to
(5), but the EVD generates orthogonal eigenvectors, a property that is not necessarily shared by the steering vectors in (5). Therefore, the eigenvectors do not provide direct access to the steering vectors and thus to the source parameters $\left\{\varphi_{i}, \vartheta_{i}\right\}$. However, if eigenvalues are arranged in descending order, the EVD provides a subspace partitioning

$$
\mathbf{R}=\left[\begin{array}{ll}
\mathbf{Q}_{\mathrm{s}} & \mathbf{Q}_{\mathrm{n}}
\end{array}\right]\left[\begin{array}{cc}
\boldsymbol{\Lambda}_{\mathrm{s}} & \mathbf{0} \\
\mathbf{0} & \boldsymbol{\Lambda}_{\mathrm{n}}
\end{array}\right]\left[\begin{array}{l}
\mathbf{Q}_{\mathrm{s}}^{\mathrm{H}} \\
\mathbf{Q}_{\mathrm{n}}^{\mathrm{H}}
\end{array}\right]
$$

where $\boldsymbol{\Lambda}_{\mathrm{n}}=\sigma_{v}^{2} \mathbf{I}$, and $\boldsymbol{\Lambda}_{\mathrm{s}} \in \mathbb{R}^{I \times I}$ contains the dominant eigenvalues and all source steering vectors lie in the signalplus-noise subspace spanned by their corresponding eigenvectors in $\mathbf{Q}_{\mathrm{s}} \in \mathbb{C}^{M \times I}$. The remaining noise-only subspace is spanned by the columns of $\mathbf{Q}_{\mathrm{n}} \in \mathbb{C}^{M \times(M-I)}$. Hence all the source steering vectors should be orthogonal to this noise-only subspace.

\section{Multiple Signal Classification}

In the multiple signal classification (MUSIC) algorithm [1], we estimate the space-time covariance matrix $\hat{\mathbf{R}}$ from the data. Due to finite sample size, such that $\mathbf{x}[n], 0 \leq n<N$, is only available for $N$ snapshots, there will be a finite approximation error $(\mathbf{R}-\hat{\mathbf{R}})$ that depends on both $\mathbf{R}$ and $N$ [14]. As in (6), we perform a subspace decomposition

$$
\hat{\mathbf{R}}=\left[\hat{\mathbf{Q}}_{\mathrm{s}} \hat{\mathbf{Q}}_{\mathrm{n}}\right]\left[\begin{array}{cc}
\hat{\boldsymbol{\Lambda}}_{\mathrm{s}} & \mathbf{0} \\
\mathbf{0} & \hat{\boldsymbol{\Lambda}}_{\mathrm{n}}
\end{array}\right]\left[\begin{array}{c}
\hat{\mathbf{Q}}_{\mathrm{s}}^{\mathrm{H}} \\
\hat{\mathbf{Q}}_{\mathrm{n}}^{\mathrm{H}}
\end{array}\right],
$$

where the columns of $\hat{\mathbf{Q}}_{\mathrm{s}}$ and $\hat{\mathbf{Q}}_{\mathrm{n}}$ span the estimated signalplus-noise and noise-only subspaces, respectively. The estimation error in $\hat{\mathbf{R}}$ will perturb these estimated subspaces w.r.t. the ground truth in (6) [15].

Given a steering vector $\mathbf{a}_{\Omega, \varphi, \vartheta}$ for a particular $\operatorname{AoA}\{\varphi, \vartheta\}$, we then test how much it leaks into the estimated noise-only subspace $\hat{\mathbf{Q}}_{\mathrm{n}}$, i.e.

$$
\xi_{\mathrm{MUSIC}}^{-1}(\varphi, \vartheta)=\left\|\hat{\mathbf{Q}}_{\mathrm{n}}^{\mathrm{H}} \mathbf{a}_{\Omega, \varphi, \vartheta}\right\|_{2}^{2} .
$$

This is repeated for each AoA $\{\varphi, \vartheta\}$ of interest. The function $\xi_{\text {MUSIC }}^{-1}(\varphi, \vartheta)$ is zero if a steering vector lies entirely outside the estimated noise-only subspace. Typically, in MUSIC, instead of looking for zeros/dips of $\xi_{\text {MUSIC }}^{-1}(\varphi, \vartheta)$, we inspect $\xi_{\operatorname{MUSIC}}(\varphi, \vartheta)$ for poles/spikes.

\section{Graph Topology And GRaph Signal Processing}

In the following, akin to [11], [12], we operate with a single source, $I=1$ (for brevity, let $\Omega_{1}=\Omega$ ), and investigate how GSP could assist us with the AoA estimation problem.

\section{A. Spatial Adjacency Matrix}

In [12], the adjacency matrix takes into account the phase shift between neighbouring nodes in a ULA, which induces sparsity. For the arbitrary 3-d array considered here, defining neighbourhood or ordering of the sensors is less straightforward, and hence we work with the cross-correlation between nodes. This leads to a fully connected graph. Since all elements have the same gain towards the source signal, they have an 
instantaneous cross-correlation of identical magnitude. Hence for a single narrowband source with frequency $\Omega$, we have

$$
\begin{aligned}
\mathbf{A}_{\mathrm{s}} & =\frac{1}{M}\left[\begin{array}{cccc}
0 & \mathrm{e}^{-\mathrm{j} \Omega \tau_{1,2}} & \ldots & \mathrm{e}^{-\mathrm{j} \Omega \tau_{1, M}} \\
\mathrm{e}^{-\mathrm{j} \Omega \tau_{2,1}} & 0 & \cdots & \mathrm{e}^{-\mathrm{j} \Omega \tau_{2, M}} \\
\vdots & & \ddots & \vdots \\
\mathrm{e}^{-\mathrm{j} \Omega \tau_{M, 1}} & \ldots & \mathrm{e}^{-\mathrm{j} \Omega \tau_{M,(M-1)}} & 0
\end{array}\right] \\
& =\mathbf{a}_{\Omega, \varphi, \vartheta} \mathbf{a}_{\Omega, \varphi, \vartheta}^{\mathrm{H}}-\frac{1}{M} \mathbf{I},
\end{aligned}
$$

where $\mathbf{a}_{\Omega, \varphi, \vartheta}$ is the steering vector defined in (2). The lag $\tau_{i, j}$ is the relative delay experienced by the source signal between the $i$ th and $j$ th sensor elements as defined in (3). The only sparsity of $\mathbf{A}_{\mathrm{S}}$ in (9) are the zero values on the diagonal. The much sparser arrangement in [12] has little consequence, since the EVD of $\mathbf{A}_{\mathrm{s}}$ generally will not reflect this sparsity.

\section{B. Temporal Adjacency Matrix}

If $N$ snapshots of $\mathbf{x}[n], n=0, \ldots(N-1)$, are available, then this temporal window of the data can be embedded into the graph structure. Similar to the cyclic graph structure for the spatial ULA component, in [12] the temporal dimension is embedded as a cyclic graph for each sensor element, such that the overall adjacency matrix emerges as a Kronecker product between the spatial and the temporal adjacency matrices.

For a narrowband signal at frequency $\Omega$, the snapshots will induce a phase progression which can be gathered in a vector $\mathbf{a}_{\Omega} \in \mathbb{C}^{N}$,

$$
\mathbf{a}_{\Omega}=\frac{1}{\sqrt{N}}\left[1, \mathrm{e}^{\mathrm{j} \Omega}, \ldots, \mathrm{e}^{\mathrm{j}(N-1) \Omega}\right]^{\mathrm{H}},
$$

that is similar to a steering vector. Thus, we formulate

$$
\mathbf{A}_{\mathrm{t}}=\mathbf{a}_{\Omega} \mathbf{a}_{\Omega}^{\mathrm{H}}-\frac{1}{N} \mathbf{I}
$$

as an $N \times N$ adjacency matrix of the temporal graph associated with each sensor. With this and (9), the overall adjacency matrix of the graph connecting all sensors in both spatial and temporal domains becomes $\mathbf{A}=\mathbf{A}_{\mathrm{s}} \otimes \mathbf{A}_{\mathrm{t}}$, with $\otimes$ denoting the Kronecker product (see Fig. 2 in [12] for the ULA example).

\section{Graph Fourier Transform}

Following [12], we use the graph Fourier transform (GFT) [18] to analyse signals defined on graphs. It is based on the EVD of the adjacency matrix $\mathbf{A}=\mathbf{A}_{\mathrm{s}} \otimes \mathbf{A}_{\mathrm{t}}$,

$$
\mathbf{A}=\mathbf{Q} \boldsymbol{\Lambda} \mathbf{Q}^{\mathrm{H}} \text {. }
$$

Note that with the EVDs $\mathbf{A}_{\mathrm{s}}=\mathbf{Q}_{\mathrm{s}} \boldsymbol{\Lambda}_{\mathrm{s}} \mathbf{Q}_{\mathrm{s}}^{\mathrm{H}}$ and $\mathbf{A}_{\mathrm{t}}=\mathbf{Q}_{\mathrm{t}} \boldsymbol{\Lambda}_{\mathrm{t}} \mathbf{Q}_{\mathrm{t}}^{\mathrm{H}}$, (12) simplifies [12] to $\boldsymbol{\Lambda}=\boldsymbol{\Lambda}_{\mathrm{s}} \otimes \boldsymbol{\Lambda}_{\mathrm{t}}$ and $\mathbf{Q}=\mathbf{Q}_{\mathrm{s}} \otimes \mathbf{Q}_{\mathrm{t}}$, whereby the latter represents the GFT matrix, containing the GFT basis in its columns [19].

For the EVD of $\mathbf{A}_{\mathrm{s}}$, given (9) it is easy to show that

$$
\mathbf{A}_{\mathrm{s}}=\left[\begin{array}{ll}
\mathbf{a}_{\Omega, \varphi, \vartheta} & \mathbf{V}_{\mathrm{s}}
\end{array}\right] \boldsymbol{\Lambda}_{\mathrm{s}}\left[\begin{array}{c}
\mathbf{a}_{\Omega, \varphi, \vartheta}^{\mathrm{H}} \\
\mathbf{V}_{\mathrm{s}}^{\mathrm{H}}
\end{array}\right]
$$

where $\boldsymbol{\Lambda}_{\mathrm{s}}=\operatorname{diag}\left\{\frac{M-1}{M},-\frac{1}{M}, \ldots,-\frac{1}{M}\right\}$. Due to the eigenvalue $\lambda_{\mathrm{s}}=-\frac{1}{M}$ possessing an algebraic multiplicity of $(M-1), \mathbf{V}_{\mathrm{s}} \in \mathbb{C}^{M \times(M-1)}$ can be selected arbitrarily with orthogonal columns such that $\mathbf{V}_{\mathrm{S}}^{\mathrm{H}} \mathbf{V}_{\mathrm{s}}=\mathbf{I}$ and $\mathbf{V}_{\mathrm{s}}^{\mathrm{H}} \mathbf{a}_{\Omega, \varphi, \vartheta}=\underline{0}$.
For the temporal component $\mathbf{A}_{\mathrm{t}}$ of the adjacency matrix, based on (11) and analogously to (13), we have that

$$
\mathbf{A}_{\mathrm{t}}=\left[\begin{array}{ll}
\mathbf{a}_{\Omega} & \mathbf{V}_{\mathrm{t}}
\end{array}\right] \boldsymbol{\Lambda}_{\mathrm{t}}\left[\begin{array}{c}
\mathbf{a}_{\Omega}^{\mathrm{H}} \\
\mathbf{V}_{\mathrm{t}}^{\mathrm{H}}
\end{array}\right],
$$

with $\boldsymbol{\Lambda}_{\mathrm{t}}=\operatorname{diag}\left\{\frac{N-1}{N},-\frac{1}{N}, \ldots,-\frac{1}{N}\right\}$ and $\mathbf{V}_{\mathrm{t}} \in \mathbb{C}^{N \times(N-1)}$ arbitrary such that $\mathbf{V}_{\mathrm{t}}^{\mathrm{H}} \mathbf{V}_{\mathrm{t}}=\mathbf{I}$ and $\mathbf{V}_{\mathrm{t}}^{\mathrm{H}} \mathbf{a}_{\Omega}=\underline{0}$.

This defines the GFT matrix $\mathbf{Q}$ in terms of the spatial and temporal steering vectors $\mathbf{a}_{\Omega, \varphi, \vartheta}$ and $\mathbf{a}_{\Omega}$, and their orthogonal complements $\mathbf{V}_{\mathrm{s}}$ and $\mathbf{V}_{\mathrm{t}}$. It is not difficult to show, when the array configuration is reduced to a ULA, that this $Q$ is identical (up to ambiguities w.r.t. $\mathbf{V}_{\mathrm{s}}$ and $\mathbf{V}_{\mathrm{t}}$ ) to the GFT associated with the sparse adjacency matrices selected in [12].

\section{MUSIC-LIKE SPECTRUM BASED ON GRAPHS AND GRAPH SignALS}

\section{A. Scanning Subspaces}

Given a fully-connected graph defined by the adjacency matrix $\mathbf{A}=\mathbf{A}_{\mathrm{s}} \otimes \mathbf{A}_{\mathrm{t}}$, we define the graph signal as the concatenation of the snapshots $\mathbf{x}[n]$, i.e.,

$$
\boldsymbol{x}^{\mathrm{H}}=\left[\mathbf{x}^{\mathrm{H}}[0], \mathbf{x}^{\mathrm{H}}[1], \ldots, \mathbf{x}^{\mathrm{H}}[N-1]\right] .
$$

In the noiseless case, this graph signal $\boldsymbol{x}$ for a source defined by $\left\{\Omega, \varphi_{1}, \vartheta_{1}\right\}$ is aligned with the principal eigenvector of the GFT matrix Q, i.e. $\mathbf{a}_{\Omega, \varphi_{1}, \vartheta_{1}} \otimes \mathbf{a}_{\Omega}$. Consequently, $\boldsymbol{x}$ is orthogonal to the space spanned by the other eigenvectors. Similar to the MUSIC algorithm, for robustness when noise is present (or for multiple sources), instead of probing for the alignment of $\boldsymbol{x}$ with this principal eigenvector, we check for the leakage of $\boldsymbol{x}$ into the complement $\mathbf{V}$ of the principal eigenvector, which is given by

$$
\mathbf{V}_{\Omega, \varphi, \vartheta}=\left[\mathbf{a}_{\Omega, \varphi, \vartheta} \otimes \mathbf{V}_{\mathrm{t}}, \quad \mathbf{V}_{\mathrm{s}} \otimes \mathbf{Q}_{\mathrm{t}}\right] .
$$

The columns of $\mathbf{V}_{\Omega, \varphi, \vartheta}$ therefore span the noise-only subspace of the graph signal. With this, we scan the graph signal for leakage into the noise-only subspace, i.e. we look for zeros/dips in

$$
\xi_{\mathrm{GSP}}^{-1}(\varphi, \vartheta)=\left\|\mathbf{V}_{\Omega, \varphi, \vartheta}^{\mathrm{H}} \boldsymbol{x}\right\|_{2}^{2},
$$

with $\Omega=\Omega_{1}$ fixed,s or akin to MUSIC, check $\xi_{\mathrm{GSP}}(\varphi, \vartheta)$ for poles/spikes.

\section{B. Comparison to MUSIC}

The approach in (17) is similar to MUSIC in (8) in the sense that a noise-only subspace is scanned. The difference lies in which quantities are estimated, and which quantities are used to scan a range of parameters. In the GSP approach for $\xi_{\mathrm{GSP}}(\varphi, \vartheta)$ in (17), the noisy data contributes the vector $\boldsymbol{x}$ while we obtain $\mathbf{V}_{\Omega, \varphi, \vartheta}$ deterministically for a range of values $\{\Omega, \varphi, \vartheta\}$. In contrast, MUSIC estimates the noise-only subspace from the (noisy) sample covariance matrix, and scans this with the deterministic steering vector $\mathbf{a}_{\Omega, \varphi, \vartheta}$.

As a further difference, the GSP approach for $\xi_{\mathrm{GSP}}(\varphi, \vartheta)$ utilises a vector space of dimension $M N$, and attempts to average out noise through the matrix multiplication in (17). 
MUSIC averages across the temporal window of $N$ snapshots to calculate an $M \times M$ sample space time covariance matrix, such that the dimension of the vector used for scanning is only $M$.

\section{Numerical Efficiency}

For both MUSIC and GSP approaches (8) and (17), we need to evaluate a norm of the form $\left\|\mathbf{V}^{\mathrm{H}} \mathbf{y}\right\|_{2}^{2}$. Therefore, we have that

$$
\begin{aligned}
\left\|\mathbf{V}^{\mathrm{H}} \mathbf{y}\right\|_{2}^{2} & =\left\|\mathbf{V} \mathbf{V}^{\mathrm{H}} \mathbf{y}\right\|_{2}^{2} \\
& =\left\|\left(\mathbf{I}-\mathbf{V}^{\perp} \mathbf{V}^{\perp, \mathrm{H}}\right) \mathbf{y}\right\|_{2}^{2},
\end{aligned}
$$

where $\left[\mathbf{V}^{\perp}, \mathbf{V}\right]$ is unitary. The first step is easy to confirm via an SVD of $\mathbf{V}$, and the second step is based on subspace projections [16].

Identifying $\mathbf{V}^{\perp}=\hat{\mathbf{Q}}_{\mathrm{s}} \in \mathbb{C}^{M \times 1}$ and $\mathbf{y}=\mathbf{a}_{\Omega, \varphi, \vartheta}$, MUSIC can thus be evaluated equivalently as

$$
\xi_{\text {MUSIC }}^{-1}(\varphi, \vartheta)=\left\|\mathbf{a}_{\Omega, \varphi, \vartheta}-\hat{\mathbf{Q}}_{\mathrm{s}}\left(\hat{\mathbf{Q}}_{\mathrm{s}}^{\mathrm{H}} \mathbf{a}_{\Omega, \varphi, \vartheta}\right)\right\|_{2}^{2} .
$$

Calculating (19) only requires $2 M$ complex multiply accumulates (MACs) per tested angle pair $\{\varphi, \vartheta\}$. However to determine $\hat{\mathbf{Q}}_{\mathrm{s}}$, MUSIC additionally requires the evaluation of $\hat{\mathbf{R}}$, which over $N$ snapshots absorbs $\frac{1}{2} M(M+1) N$ MACs, and the calculation of its EVD at a cost of $\mathcal{O}\left(M^{3}\right)$.

For the GSP approach, in (18) we identify $\mathbf{V}^{\perp}=\mathbf{a}_{\Omega, \varphi, \vartheta} \otimes$ $\mathbf{a}_{\Omega}$ and $\mathbf{y}=\boldsymbol{x}$, such that instead of (17), we can evaluate

$$
\xi_{\mathrm{GSP}}^{-1}(\varphi, \vartheta)=\left\|\mathbf{x}-\left(\mathbf{a}_{\Omega, \varphi, \vartheta} \otimes \mathbf{a}_{\Omega}\right)\left(\mathbf{a}_{\Omega, \varphi, \vartheta} \otimes \mathbf{a}_{\Omega}\right)^{\mathrm{H}} \boldsymbol{x}\right\|_{2}^{2} .
$$

This requires $2 M N$ MACs for the evaluation of an angle pair $\{\varphi, \vartheta\}$, but no cost needs to be expended on covariance estimation or an EVD.

\section{Simulations and Results}

To compare the GSP approach and MUSIC, we assume an array of $M=5$ sensors that lies within a cube of unit side length, where unity refers to critical spatial sampling at $\Omega=\pi$. The three spatial coordinates for each sensor are drawn-once for all simulations-from a uniform distribution $\mathcal{U}(0,1)$, while the source signal at a narrowband frequency $\Omega_{1}=\frac{\pi}{2}$ illuminates the array from an azimuth $\varphi_{1}=20^{\circ}$ and an elevation $\vartheta_{1}=70^{\circ}$. As in [12], we collect $N=41$ snapshots of data at an adjustable signal-to-noise ratio (SNR).

Over a grid of azimuth and elevations angles $\{\varphi, \vartheta\}$, Figs. 1 to 4 show the evaluations of (19) and (20) for $\Omega=\Omega_{1}$ and two different SNR regimes. For the high SNR scenario of $30 \mathrm{~dB}$ in Figs. 1 and 2, both the GSP approach and MUSIC provide an accurate extremum at the source parameters $\left\{20^{\circ}, 70^{\circ}\right\}$, with a sharper lobe for MUSIC. For the lower SNR scenario of $0 \mathrm{~dB}$ in Figs. 3 and 4, the peaks are of lower intensity compared to the $30 \mathrm{~dB}$ scenario, with $\xi_{\mathrm{MUSIC}}(\varphi, \vartheta)$ still providing a sharper peak compared to $\xi_{\mathrm{GSP}}(\varphi, \vartheta)$.

To assess the accuracy of the proposed GSP-based method, we evaluate the estimated AoA using the cost functions in (19) and (20) as

$$
\left\{\hat{\varphi}_{1}, \hat{\vartheta}_{1}\right\}=\arg \min _{\varphi, \vartheta} \xi_{i}^{-1}(\varphi, \vartheta)
$$

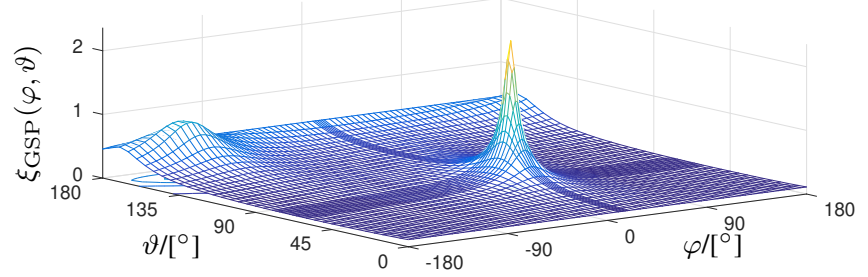

Fig. 1. GSP-based metric $\xi_{\mathrm{GSP}}(\varphi, \vartheta)$ for $M=5$ and $N=41$ at $30 \mathrm{~dB}$ SNR.

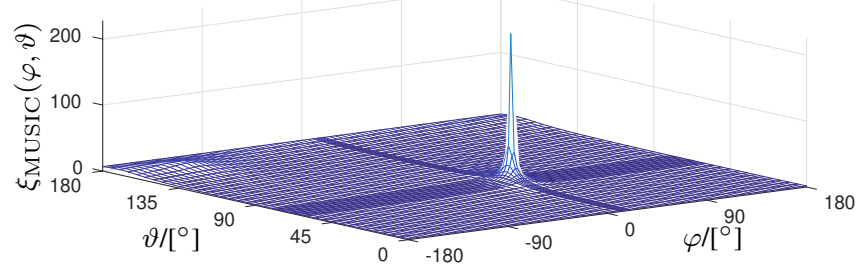

Fig. 2. MUSIC metric $\xi_{\operatorname{MUSIC}}(\varphi, \vartheta)$ for $M=5$ and $N=41$ at $30 \mathrm{~dB}$ SNR.

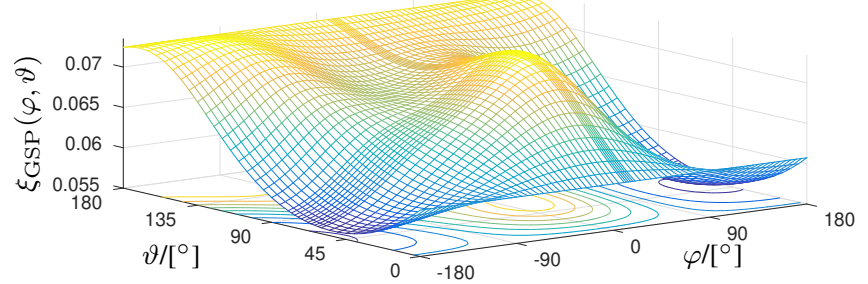

Fig. 3. GSP-based metric $\xi_{\mathrm{GSP}}(\varphi, \vartheta)$ for $M=5$ and $N=41$ at $0 \mathrm{~dB}$ SNR.

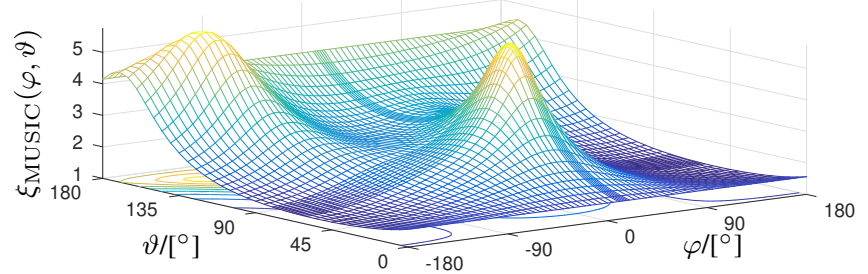

Fig. 4. MUSIC metric $\xi_{\operatorname{MUSIC}}(\varphi, \vartheta)$ for $M=5$ and $N=41$ at $0 \mathrm{~dB}$ SNR.

with $i=\{$ MUSIC, GSP $\}$. This non-linear optimisation problem is solved by the simplex algorithm [17], and performed over an ensemble of $10^{4}$ realisation with different noise vectors $\mathbf{v}[n]$ in (4) for every SNR value under test. As a metric, we measure the angle between the direction $\mathbf{k}_{\varphi_{1}, \vartheta_{1}}$ of the source, and the direction associated with the estimated source parameters, $\mathbf{k}_{\hat{\varphi}_{1}, \hat{\vartheta}_{1}}$ via

$$
\psi=\angle\left\{\mathbf{k}_{\varphi_{1}, \vartheta_{1}}, \mathbf{k}_{\hat{\varphi}_{1}, \hat{\vartheta}_{1}}\right\}=\arccos \left(\mathbf{k}_{\varphi_{1}, \vartheta_{1}}^{\mathrm{H}} \mathbf{k}_{\hat{\varphi}_{1}, \hat{\vartheta}_{1}}\right)
$$

i.e. the Hermitian angle between the two direction vectors.

Varying the SNR over the range from $-20 \mathrm{~dB}$ to $20 \mathrm{~dB}$, mean values of $\psi$ for the GSP-based approach and for MUSIC are shown in Fig. 5. For high SNR, as expected from the surface of the metrics in Figs. 1 and 2, the mean performance is identical. To give an insight into the spread of the distribution of the ensemble of $10^{4}$ experiments per SNR, Fig. 5 also contains the quartiles, which highlight that the MUSIC and GSP provide asymptotically identical accuracy as the SNR increases. 


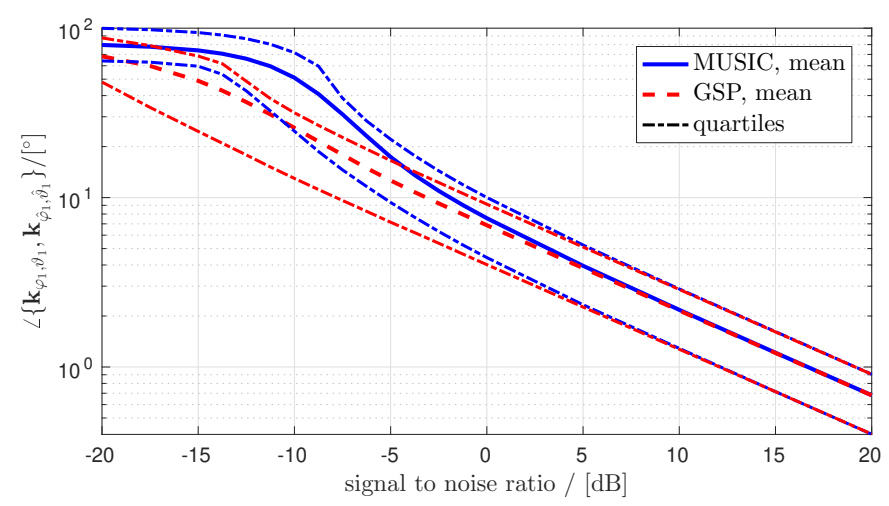

Fig. 5. Ensemble results for measuring the deviation of the estimated direction from the true one, $\psi$ in (22), in dependency of the SNR of the data.

Towards low SNRs, the covariance matrix estimate $\hat{\mathbf{R}}$ becomes increasingly poor, leading to larger perturbation of the subspaces in (7) as compared to (6). For MUSIC, this subspace leakage is known to reach a cliff-edge type performance degradation as the estimation error increases [3]. This degradation can be observed in Fig. 5 for SNR region below $0 \mathrm{~dB}$; similar results have been observed in e.g. [4]. Albeit that neither algorithms produce very accurate results, the GSPbased approach degrades gracefully and hence at lower SNR values outperforms MUSIC, since (20) essentially represents a matched filter using a beamformer [13], which is known to be optimal for the single user case.

For the above simulations, solving (21) under Matlab on an Intel CPU N3350 1.1GHz with 4GB RAM takes on average $11.9 \mathrm{~ms}$ CPU time for the GSP approach, while MUSIC takes $11.0 \mathrm{~ms}$. Since for the selected parameters $M N \approx \mathcal{O}\left(M^{3}\right)$, the two computational costs from Sec. IV-C are in balance.

\section{CONCLUSIONS}

In this paper we have investigated a GSP approach for angle of arrival estimation. Starting from [12], we have extended the approach from a ULA to an arbitrary sensor array, investigated the similarities and differences to the MUSIC algorithm, and provided a numerically efficient approach over [12] to the evaluation of both the GSP approach and MUSIC: while MUSIC estimates the noise-only subspace from data, and probes it with accurate steering vectors, in the GSP approach, the subspace is obtained from the adjacency matrix and therefore deterministically from the graph topology, which is probed by the potentially noisy graph signal.

For the selected simulation scenario and its parameters, both algorithms were of similar computational complexity. Simulations suggest that as SNR increases, the GSP approach asymptotically performs like MUSIC. At low SNR however, the GSP approach, implementing an optimum single user matched filter, avoids the fast degradation that is associated with MUSIC due to subspace leakage. This implies that the GSP approach, acting as a matched subspace detector, cannot provide any new gains for AoA estimation. This is perhaps unsurprising since GSP draws its advantages from additional information [18], while the matched subspace detector already operates optimally on all available data.

Because of the arbitrary three-dimensional array configuration, we have not embedded any measure of physical adjacency of sensors in the graph topology, and both the graph and its adjacency matrix were selected non-sparse. While this does not impact on the GFT and hence the results in this paper, sparsity in the adjacency matrix can be important when seeking distributed implementations [20] and will therefore be worth investigating as a future step.

\section{ACKNOWLEDGEMENT}

We would like to thank Geert Leus of TU Delft, The Netherlands, for very helpful discussions and input.

\section{REFERENCES}

[1] R.O. Schmidt, "Multiple emitter location and signal parameter estimation," IEEE Trans. Antennas \& Propagation, 34(3):276-280, Mar. 1986.

[2] S. Weiss, M. Alrmah, S. Lambotharan, J. McWhirter, and M. Kaveh, "Broadband angle of arrival estimation methods in a polynomial matrix decomposition framework," in IEEE CAMSAP, pp. 109-112, Dec. 2013.

[3] M. Shaghaghi and S.A. Vorobyov, "Subspace leakage analysis and improved DoA estimation with small sample size," IEEE Trans. Signal Processing, 63(12):3251-3265, June 2015.

[4] M.W. Morency, S.A. Vorobyov, and G. Leus, "Joint detection and localization of an unknown number of sources using the algebraic structure of the noise subspace," IEEE Trans. Signal Processing, 66(17):4685-4700, Sep. 2018.

[5] F. Marvasti, "Nonuniform sampling theorems for bandpass signals at or below the Nyquist density," IEEE Trans. Signal Processing, 44(3):572576, Mar. 1996.

[6] D.L. Donoho, "Compressed sensing," IEEE Trans. Information Theory, 52(4):1289-1306, Apr. 2006.

[7] H.L. Van Trees, Detection, Estimation and Modulation Theory: Optimum Array Processing. New York: Wiley, 2002.

[8] W. Liu and S. Weiss, Wideband Beamforming - Concepts and Techniques. Wiley, 2010.

[9] A. Alzin, F. Coutts, J. Corr, S. Weiss, I.K. Proudler, and J.A. Chambers, "Adaptive broadband beamforming with arbitrary array geometry," in IET/EURASIP ISP, London, UK, Dec. 2015.

[10] A. Ortega, P. Frossard, J. Kovacevic, J. M. F. Moura, and P. Vandergheynst, "Graph Signal Processing: Overview, Challenges, and Applications," Proc. of the IEEE, 106(5):808-828, May 2018.

[11] E. Alcantara, L. Atlas, and S. Abadi, "Graph signal smoothness for direction-of-arrival estimation of source targets using non-uniform line arrays," J. Acoustical Society of America, 146(4):2886-2886, 2019.

[12] L.A.S. Moreira, A.L.L. Ramos, M.L.R. de Campos, J.A. Apolinário, and F. G. Serrenho, "A graph signal processing approach to direction of arrival estimation," in European Signal Processing Conference, La Coruña, Spain, Sep. 2019.

[13] L. L. Scharf and B. Friedlander, "Matched subspace detectors," IEEE Trans. Signal Processing, 42(8):2146-2157, Aug. 1994.

[14] C. Delaosa, J. Pestana, N.J. Goddard, S. Somasundaram, and S. Weiss, "Sample space-time covariance matrix estimation," in ICASSP, Brighton, UK, pp. 8033-8037, May 2019.

[15] C. Delaosa, F.K. Coutts, J. Pestana, and S. Weiss, "Impact of space-time covariance estimation errors on a parahermitian matrix EVD," in IEEE SAM, Sheffield, UK, July 2018.

[16] G.H. Golub and C.F. Van Loan, Matrix Computations, 3rd ed. Baltimore, ML: John Hopkins University Press, 1996.

[17] J.A. Nelder and R. Mead, "A Simplex Method for Function Minimization," The Computer Journal, 7(4):308-313, Jan. 1965.

[18] D. Shuman, S. Narang, P. Frossard, A. Ortega, and P. Vandergheynst, "The emerging field of signal processing on graphs: Extending highdimensional data analysis to networks and other irregular domains," IEEE Signal Processing Magazine, 30(3):83-98, May 2013.

[19] A. Sandryhaila and J.M.F. Moura, "Discrete signal processing on graphs, IEEE Trans. Signal Processing, 61(7):1644-1656, Apr. 2013.

[20] M. Coutino, E. Isufi, and G. Leus, "Advances in distributed graph filtering," IEEE Trans. Signal Processing, 67(9):2320-2333, May 2019. 